\title{
Fabrication and Performance Test of Fresnel Zone Plate with 35 nm Outermost Zone Width in Hard X-Ray Region
}

\author{
Yoshio Suzuki, ${ }^{1}$ Akihisa Takeuchi, ${ }^{1}$ Hisataka Takenaka, ${ }^{2}$ and Ikuo Okada ${ }^{2}$ \\ ${ }^{1}$ Japan Synchrotron Radiation Research Insitute (JASRI)/SPring-8, Sayo Hyogo 679-5198, Japan \\ ${ }^{2}$ NTT-AT Nanofabrication Corporation, Atsugi, Kanagawa 243-0018, Japan
}

Correspondence should be addressed to Yoshio Suzuki, yoshio@spring8.or.jp

Received 29 October 2009; Accepted 17 June 2010

Academic Editor: Ali Khounsary

Copyright (C 2010 Yoshio Suzuki et al. This is an open access article distributed under the Creative Commons Attribution License, which permits unrestricted use, distribution, and reproduction in any medium, provided the original work is properly cited.

\begin{abstract}
A Fresnel zone plate (FZP) with $35 \mathrm{~nm}$ outermost zone width has been fabricated and tested in the hard X-ray region. The FZP was made by electron beam lithography and reactive ion etching technique. The performance test of the FZP was carried out by measuring the focused beam profile for coherent hard X-ray beam at the beamline 20XU of SPring- 8 . The full width at half maximum of the focused beam profile measured by knife-edge scan method is $34.9 \pm 2.7 \mathrm{~nm}$, that agrees well with the theoretical value of diffraction-limited resolution. Applications to scanning microscopy were also carried out.
\end{abstract}

\section{Introduction}

Many types of optical elements for X-ray microfocusing and microimaging in the hard X-ray regions have been developed. Fresnel zone plates [1], total reflection mirrors [2], multilayer mirrors [3], refractive lenses [4], and multilayer Laue lenses [5] were fabricated and applied to microfocusing of synchrotron radiation X-rays. At present, the Fresnel zone plate (FZP) manufactured by electron beam lithography is one of the best optical elements for X-ray microscopy because of its high spatial resolution (better than $50 \mathrm{~nm}$ ), negligibly small coma and small field distortion. The chromatic aberrations of FZP are usually ignorable in the hard X-ray region, because the crystal monochromators used in the synchrotron radiation beamlines produce sufficient monochromaticity for hard $\mathrm{X}$-rays $(\lambda / \Delta \lambda \sim 10000)$. Thus, the spatial resolution of FZP optics is simply determined by the numerical aperture and by the accuracy of zone structure.

Spatial resolution of about $30 \mathrm{~nm}$ is already achieved with conventional FZPs. However, these results are carried out using third-order diffraction of FZP with an outermost zone width of $50 \mathrm{~nm}[1,6]$. Although a gold zone plate with $24 \mathrm{~nm}$ outermost zone width is also fabricated and tested in hard X-ray region [7], its spatial resolution has not been reported. Some X-ray focusing optics of spatial resolution better than $30 \mathrm{~nm}$ is already reported [2, 5].
However, they are still one-dimensional focusing, and these optical elements, the multilayer Laue lenses, or the aspherical Kirkpatrick-Baez mirrors are not image-forming optics. The Fresnel zone plate in the hard X-ray region is an almost ideal image-forming optical devise up to a spatial resolution of around $10 \mathrm{~nm}[8,9]$. Therefore, the conventional FZP is still indispensable for hard X-ray microscopy. In this report, recent development of FZP with an outermost zone width of $35 \mathrm{~nm}$ is presented, and characterization of the FZP in the hard X-ray region is described.

\section{Fresnel Zone Plate Fabrication}

The FZP was fabricated at NTT-AT Nanofabrication by electron beam lithography and reactive ion etching technique. The fabrication process of FZP is shown in Figure 1. The substrate is composed of $2 \mu \mathrm{m}$ silicon nitride ( $\mathrm{SiN}$ ) membrane deposited on a Si wafer by low pressure chemical vapor deposition (CVD) method. Tantalum film with a thickness of $0.175 \mu \mathrm{m}$ was deposited by rf-sputtering method, and $\mathrm{SiO}_{2}$ film was deposited by electron cyclotron resonance (ECR) plasma CVD method on the Ta layer as an etching mask of Ta. The FZP patterns were written by point electron-beam exposure method. The $\mathrm{SiO}_{2}$ film was etched with resist mask by the reactive ion etching, and the Ta film was dry-etched with $\mathrm{SiO}_{2}$ mask by an ECR ion-stream etching. Finally, the 


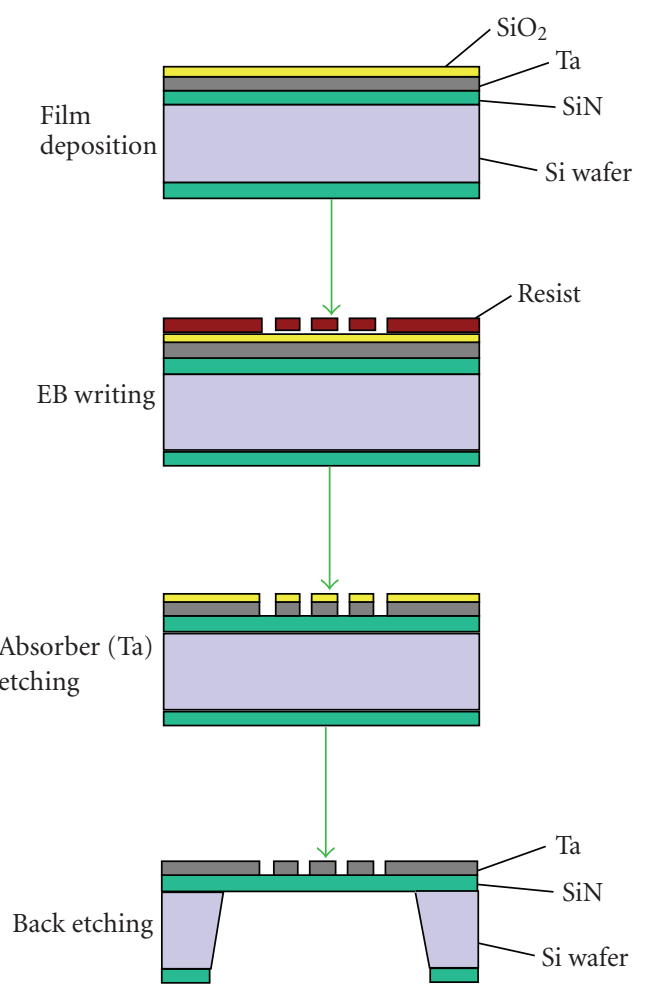

Figure 1: Processing step of Fresnel zone plate manufacturing.

Si wafer was back etched with $\mathrm{KOH}$ solution for windowing. The details of fabrication method of tantalum zone plates at the NTT-AT Nanofabrication are already reported elsewhere $[10,11]$.

Schematic drawing of the FZP structure and its SEM image is shown in Figure 2. The design width of outermost zone that determines the diffraction-limited resolution is $35 \mathrm{~nm}$. The SEM image shows a cross section of outermost zone structure that was prepared from the duplicated FZP. Fine zone structures of $35 \mathrm{~nm}$ width are precisely formed as shown in the figure. The zone pattern is designed by the simple formula of FZP as

$$
r_{n}^{2}=n \lambda f
$$

where $n$ is integer of zone number, $\lambda$ is an $\mathrm{X}$-ray wave length, and $f$ is focal length. We used a parameter set of $\lambda$ $=0.155 \mathrm{~nm}(h \nu=8 \mathrm{keV})$ and $f=40 \mathrm{~mm}$. Comparing with the spherical-aberration-corrected exact equation of zone pattern for focusing a plane wave,

$$
r_{n}^{2}=n \lambda f+\frac{n^{2} \lambda^{2}}{4}
$$

it is required that $f$ is much greater than $n \lambda / 4$ for the ignorable spherical aberrations [12]. According to the Rayleigh's quarter wavelength rule [13], the spherical aberration can be ignored, when the following condition is satisfied [8]:

$$
\frac{N^{2}}{2}<\frac{f}{\lambda}
$$

Here, $N$ is total number of zone. In order to satisfy the Rayleigh's $\lambda / 4$ rule, we chose $N=1265$, that is, $r_{N}=88.56 \mu \mathrm{m}$ (diameter of $177.12 \mu \mathrm{m}$ ). Then, the outermost zone width becomes $35.00 \mathrm{~nm}$. The $\lambda / 4$ rule is satisfied in the wavelength region shorter than $2.8 \mathrm{~nm}$. Thus, the spherical aberration can be ignored in the hard X-ray region.

The theoretical spatial resolution (Rayleigh resolution limit) for the first-order diffraction of FZP is known to be $1.22 d_{N}$, where $d_{N}$ is the width of outermost zone [12]. Therefore, if the zone structure is ideal, spatial resolution of the FZP could be $43 \mathrm{~nm}$. It should be noted that the knifeedge scan method, which is generally used for measuring the focused beam shape, gives a line spread function of beam profile. The line-spread-function, $\operatorname{LSF}(x)$, of a diffractionlimited focal spot for circular aperture lens is given by onedimensional integral of point spread function as

$$
\operatorname{LSF}(x)=\int_{-\infty}^{\infty} \frac{\left(2 J_{1}\left(\sqrt{x^{2}+y^{2}}\right)\right)^{2}}{x^{2}+y^{2}} d y,
$$

where $J_{1}(x)$ is a Bessel function of the first kind. The Rayleigh's criterion for spatial resolution is defined by the first minimum of the Bessel function as $J_{1}\left(x_{1}\right)=0$, and the solution of $x_{1}=1.220 \pi$ is usually used as a definition of diffraction-limited resolution [13], while the full width at half maximum (FWHM) of point-spreadfunction, $\left\{2 J_{1}(x) / x\right\}^{2}$, is $1.0290 \pi$. It is shown by the numerical calculation of the above formula that the FWHM of line-spread-function is given by $x=1.0016 \pi$. Therefore, the FWHM of line-spread-function is nearly equal to the outermost zone width of ideal FZP, and the theoretical FWHM of knife-edge scan profile for the diffraction-limited micro focus beam is $1.0016 d_{N}$.

The calculated diffraction efficiency for the $0.175 \mu \mathrm{m}$ thick tantalum FZP is only $0.8 \%$ for $8 \mathrm{keV}$ X-rays. This low efficiency may sometimes be a problem for nanobeam applications or for imaging microscopy because of low signal intensity. However, the low diffraction efficiency can be compensated by high brilliance X-ray sources. Some scanning microscopy experiments can be carried out with this zone plate, and the application to the imaging microscopy would also be capable at the expense of relatively long exposure time and high radiation dose to specimen. This low diffraction efficiency is not a problem for characterization of FZP.

The thickness of FZP $(0.175 \mu \mathrm{m})$ is, however, not optimized for $8 \mathrm{keV} \mathrm{X}$-rays. The optimum thickness of Ta zone plate for $8 \mathrm{keV}$ is $1.9 \mu \mathrm{m}$. Therefore, higher aspect ratio of zone structure is required for improvement of diffraction efficiency. The aspect ratio of tantalum one-to-one zone structure is presently limited by technological reason, mainly for reactive dry-etching process. The present limit of aspect ratio is about 10 . The aspect ratio doubling by stacking two zone plates [7] would be a promising method for higher diffraction efficiency. Zone material of high electron density is adequate for X-ray zone plates, because the optimized thickness is in proportion to the reciprocal of electron density. Gold is generally chosen as the zone material for its high density and fabrication technology, for example, electroplating of gold. The density of tantalum is less than 


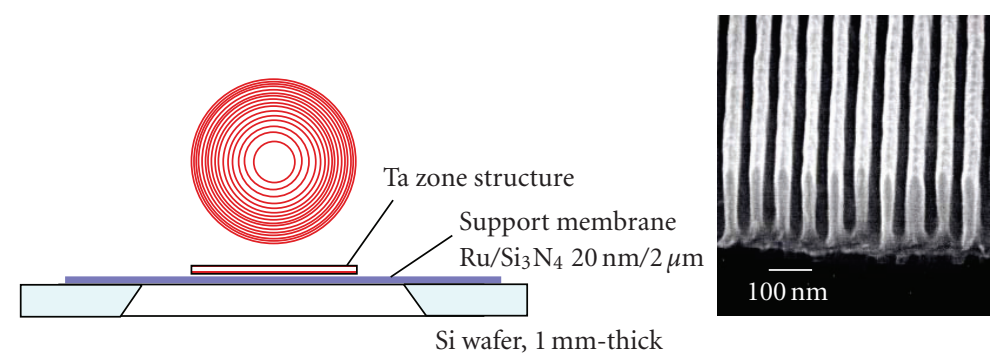

FIGURE 2: Schematic drawing and cross-sectional SEM image of Fresnel zone plate fabricated by electron beam lithography method. Width of outermost zone is $35 \mathrm{~nm}$.

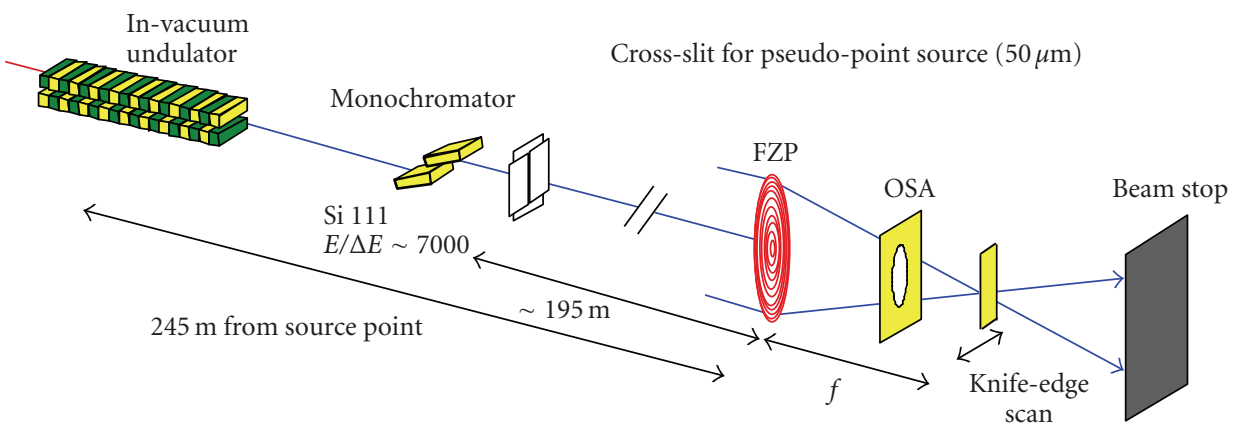

FIGURE 3: Experimental setup for X-ray nanofocusing at the beamline 20XU of SPring-8. FZP: Fresnel zone plate. OSA: order selecting aperture, a pinhole with $20 \mu \mathrm{m}$-diameter made of $0.3 \mathrm{~mm}$-thick platinum plate. Beam Stop: direct beam stop for dark-field edge-scan, tantalum 3 mm-thick plate.

that of gold by about $14 \%$. Therefore, the zone plate made of Ta usually gives lower efficiency than that made of Au in the case of same zone thickness. However, we consider that the difference of diffraction efficiency is not significant, and the tantalum is also one of the best materials for hard X-ray zone plates. In some cases, for example, near the absorption edge of gold, tantalum zone plate with the same thickness can give slightly higher efficiency than gold zone plate.

\section{Performance Test of FZP}

Performance test of the FZP as a nanofocusing optics has been carried out at the beamline 20XU [14] of SPring8. A schematic view of the experimental setup is shown in Figure 3. An X-ray energy of $8 \mathrm{keV}$ was chosen in the experiment. The energy resolution of monochromator is about $1.1 \mathrm{eV}$ for $8 \mathrm{keV} \mathrm{X}$-rays. To avoid the chromatic aberration of FZP optics, the relative spectral bandwidth of incident $\mathrm{X}$-ray beam, $\Delta \lambda / \lambda$, must be less than or equal to the inverse of total number of zone [12], as

$$
\frac{\Delta \lambda}{\lambda} \leq \frac{1}{N}
$$

Comparing with the zone number of the FZP, $N=1265$, the monochromaticity of $\Delta E / E \sim 1 / 7000$ is sufficient for testing the performance of FZP.

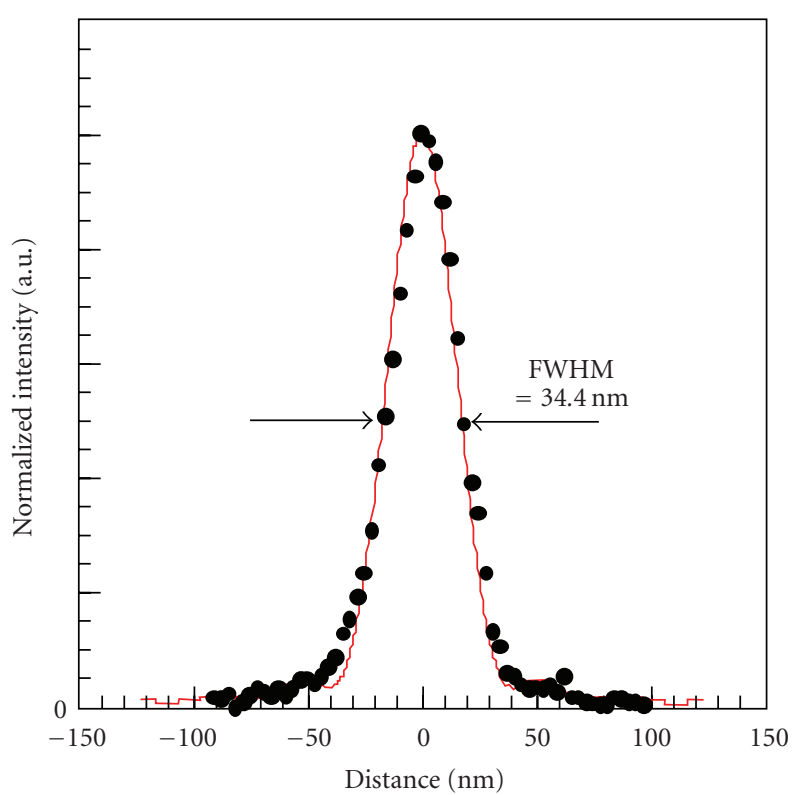

Figure 4: Focused beam profile measured by dark-field edge scan. Edge scan was carried out with $3.125 \mathrm{~nm}$ step and $1 \mathrm{~s}$ integration time per point. X-ray energy is $8 \mathrm{keV}$. An edge of tantalum thin film $(0.5 \mu \mathrm{m}$-thick $)$ is used as a knife-edge. Solid circles are measured data, and the red line represents theoretical profile for ideal circular aperture lens. Signal current of ionization chamber is about $2 \mathrm{pA}$ at the peak. 


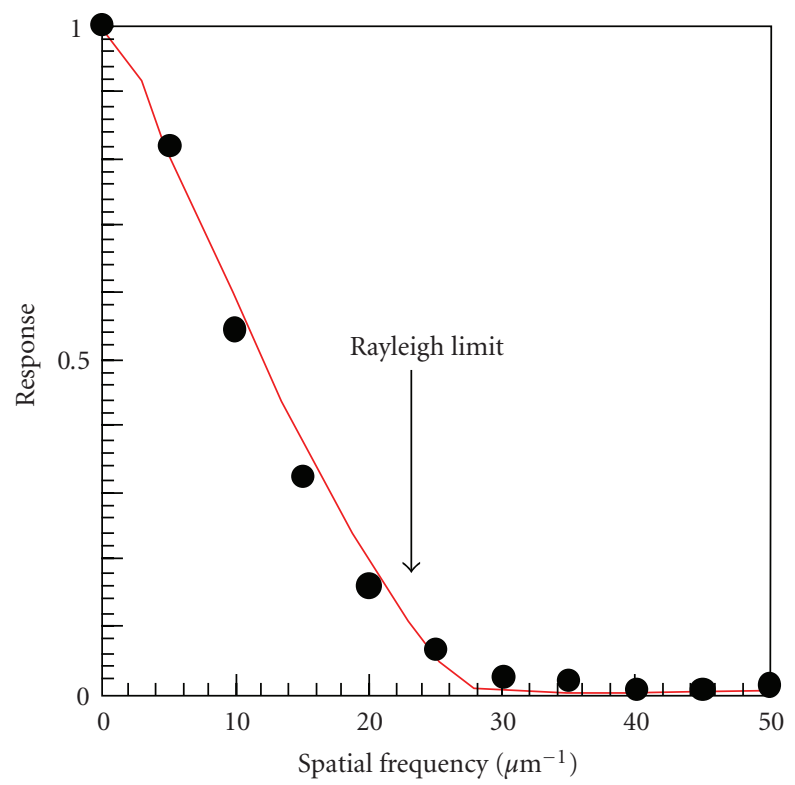

Figure 5: Modulation transfer function. Solid circles are MFT calculated from the measured beam profile shown in Figure 4. Red line is theoretical MTF obtained by numerical calculation of line-spread function.

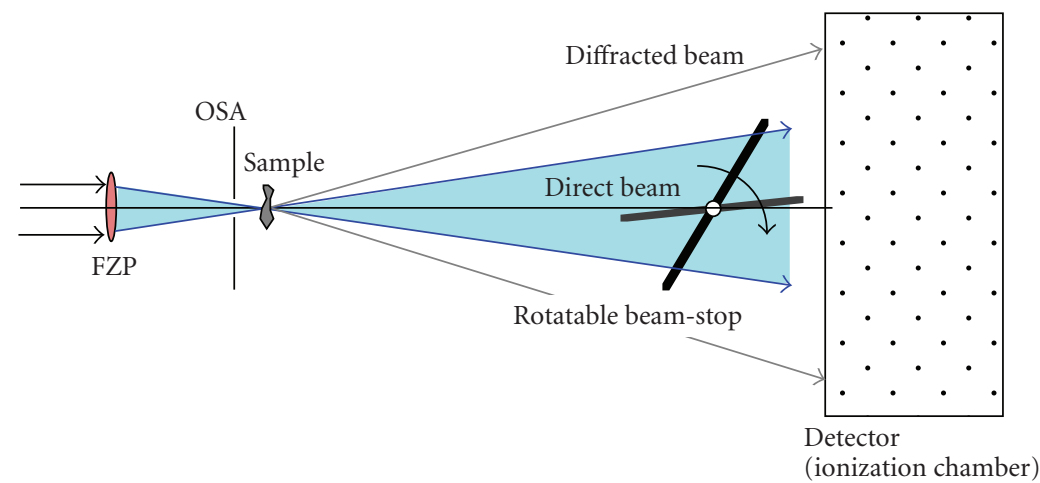

FIGURE 6: Schematic view of scanning microscopy setup for bright and dark field method. A lead thin plate (0.5 mm) beam stop is mounted on a rotating stage, and bright-field and dark-field imaging can be exchanged by rotating the beam stop.

The monochromatic beam is transported through the 200-m-long vacuum duct and is extracted in to the air at an end experimental station located at approximately $245 \mathrm{~m}$ from the light source. A precise cross slit located $49 \mathrm{~m}$ from the source is used as a pseudopoint source for determining the spatial coherence at the end station. The cross slit with an opening of $50 \mu \mathrm{m} \times 50 \mu \mathrm{m}$ was used in the present experiment. Therefore, the geometrical spot size for an FZP with a focal length of $40 \mathrm{~mm}$ becomes $10 \mathrm{~nm}$ that is much less than the diffraction-limited resolution of the FZP. There are no optical elements between the cross-slit virtual source and the experimental station, except for a beamline X-ray window made of $125 \mu \mathrm{m}$-thick polyimide.

The focus spot profile is evaluated by knife-edge scan method. However, it is difficult to fabricate precise knifeedge for characterization of nano-focusing beam, because of high transmissivity of hard X-ray beam. So, the conventional knife-edge test cannot be used for the present experiment.
We employed dark-field method for the knife-edge test [1]. A pattern edge of tantalum resolution test chart is used as a knife-edge. The thickness of tantalum test pattern is $0.5 \mu \mathrm{m}$, which corresponds to an optical path difference of about $\lambda / 8$ at an X-ray wavelength of $0.155 \mathrm{~nm}$. The intensity of the diffracted wave takes its maximum at an optical thickness of $\lambda / 2$ for phase edge. Therefore, from point of view of signal intensity, the optimum thickness of knife-edge for dark-field method is $\lambda / 2$. However, the thickness of $\lambda / 8$ is still sufficient for the beam profile measurement. The thicker knife-edge may give higher dark-field signal, but the thickness must be much less than the depth of focus that is $7.9 \mu \mathrm{m}$ in the present experimental condition. The test pattern edge was set parallel to the optical axis in order to avoid broadening of edge-scan profiles. The misalignment of substrate of test pattern was within an angle of about 0.3 degree.

The knife-edge scanning was carried out using a conventional mechanical stage driven by an open-loop-controlled 

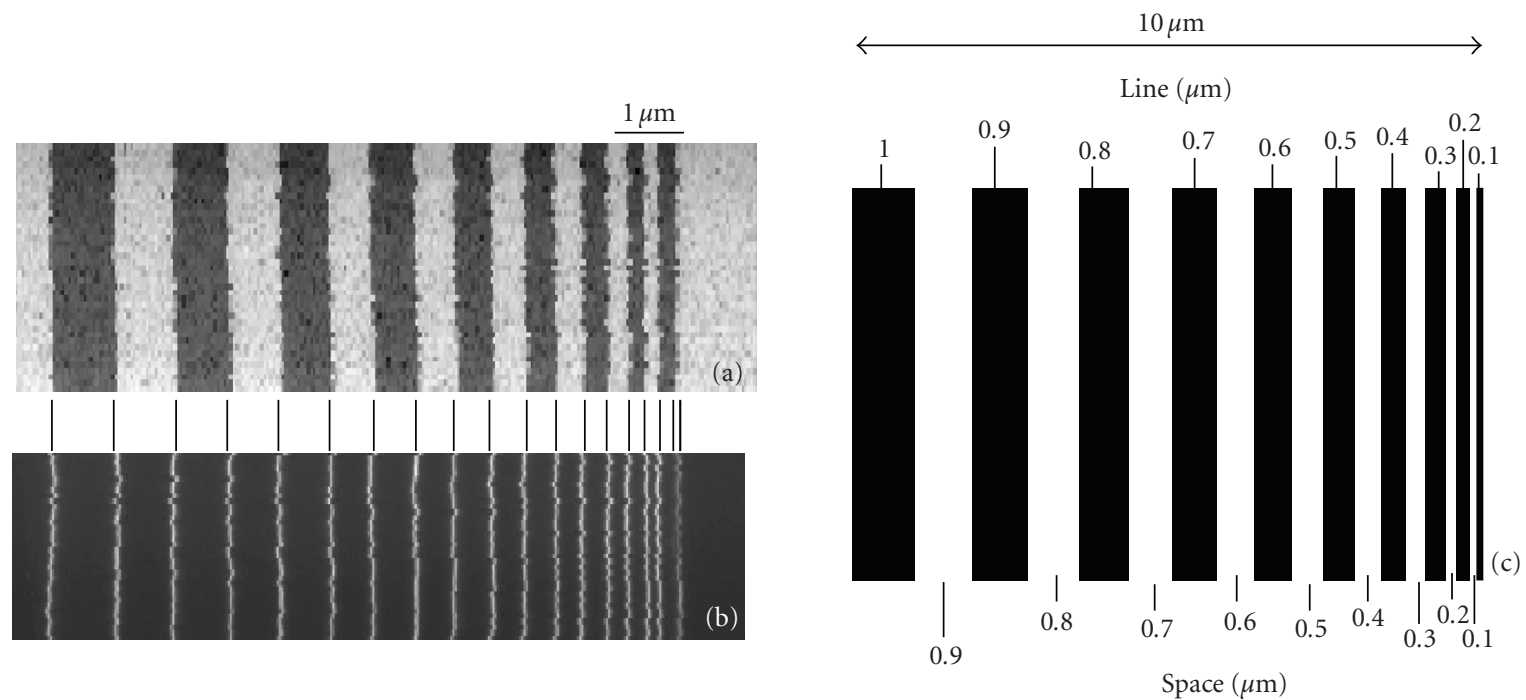

FIGURE 7: Scanning microscopy images of line and space test patterns. X-ray energy is $8 \mathrm{keV}$. (a) Bright field image, $50 \mathrm{~nm}$ pixel size and $0.2 \mathrm{~s}$ dwell time. (b) Dark field image, $12.5 \mathrm{~nm}$ pixel size and $0.2 \mathrm{~s}$ dwell time. (c) Schematic drawing of line and space patterns. Black area corresponds to tantalum-deposited regions.

step motor. The accuracy of the translation stage is measured

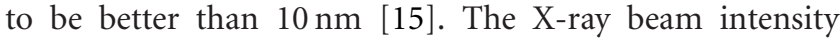
was measured with ionization chamber detectors with $14 \mathrm{~cm}$ electrode length. One is an air-flowed ionization chamber placed in front of the FZP, and the other is an argon-flowed ionization chamber behind the knife-edge. A beam stop is placed in front of the argon-flowed ionization chamber for the purpose of dark-field edge scan.

A typical example of measured beam profiles is shown in Figure 4. The FWHM of the focused beam profile shown in Figure 4 is $34.4 \mathrm{~nm}$. The accuracy of beam size measurement was estimated from repeatability of measured beam size. The average of thirteen measurements around the focus is $34.9 \mathrm{~nm}$, and the error of beam size measurement is $2.7 \mathrm{~nm}$ in standard deviation. Therefore, it is concluded that the focused beam size is $34.9 \mathrm{~nm} \pm 2.7 \mathrm{~nm}$ in FWHM. As shown in the figure, the measured beam profile agrees well with the theoretical one. It is concluded that nanometer-resolution imaging is capable in the hard X-ray region by using the FZP as a beam-focusing device or as an objective lens for imaging microscopy. The measured diffraction efficiency of the FZP is $0.7 \%$ for $8 \mathrm{keV} \mathrm{X}$-rays. This value agrees well with the theoretical efficiency $(0.8 \%)$ of $0.175 \mu \mathrm{m}$-thick Ta zone plate. Modulation transfer function (MTF) obtained from the measured beam profile in Figure 4 is shown in Figure 5. The Rayleigh's resolution limit $\left(1.22 d_{N}\right)$ for $35 \mathrm{~nm}$ outermost zone width that corresponds to a spatial frequency of $23.4 \mu \mathrm{m}^{-1}$ is indicated by an arrow in the figure. The measured MTF at $23.4 \mathrm{cycle} / \mu \mathrm{m}$ is about $10 \%$.

\section{Scanning Microscopy Experiments}

Experimental setup for scanning microscopy experiment is shown in Figure 6. Bright field imaging and dark-field imaging [16] can be exchanged by rotating the beam stop in front of the detector. The beam stop is made of a lead plate with a thickness of $0.5 \mathrm{~mm}$. Scanning microscopy images of a test object are shown in Figures 7(a) and 7(b). The specimen is resolution test patterns made of $0.5 \mu \mathrm{m}$-thick tantalum that were also fabricated at NTT-AT Nanofabrication by the same process as that of the FZP. The design feature of line and space patterns is shown in Figure 7 (c). The fine patterns up to $0.2 \mu \mathrm{m}$ were clearly seen in the measured images. The beam flux at the sample position was about $4 \times 10^{6}$ photons $/ \mathrm{s}$, and signal intensity in dark field mode was $2 \times 10^{5}$ photons/s.

Although the design of test pattern has the $0.1 \mu \mathrm{m}$ wide line and space patterns, the $0.1 \mu \mathrm{m}$ wide patterns are not observed in the measure images, and a weak line is recognized at the right edge of pattern in the dark-field image where the $0.1 \mu \mathrm{m}$-wide line should be seen. The right edge of the $0.2 \mu \mathrm{m}$-line pattern is not observed in the darkfield image. In addition, in the bright-field image, there is a faint contrast pattern at the right side of the $0.2 \mu \mathrm{m}$-line pattern. From these results, we consider that the $0.1 \mu \mathrm{m}$ wide pattern is provably collapsed or slanted and contacts to the neighboring $0.2 \mu \mathrm{m}$-wide line pattern. The $0.1 \mu \mathrm{m}$-wide line and space patterns are unfortunately imperfect in the measured portion of large test patterns.

\section{Acknowledgment}

The experiments were performed at the BL20XU in SPring8 under the approval of the Japan Synchrotron Radiation Research Institute (JASRI) (Proposal no. 2006A1562).

\section{References}

[1] Y. Suzuki, A. Takeuchi, H. Takano, and H. Takenaka, "Performance test of fresnel zone plate with $50 \mathrm{~nm}$ outermost zone width in hard X-ray region," Japanese Journal of Applied Physics. Part 1, vol. 44, no. 4 A, pp. 1994-1998, 2005. 
[2] H. Mimura, H. Yumoto, S. Matsuyama et al., "Efficient focusing of hard x rays to $25 \mathrm{~nm}$ by a total reflection mirror," Applied Physics Letters, vol. 90, no. 5, Article ID 051903, 3 pages, 2007.

[3] O. Hignette, P. Cloetens, C. Morawe et al., "Nanofocusing at ESRF using graded multilayer mirrors," in Synchrotron Radiation Instrumentation: 9th International Conference on Synchrotron Radiation Instrumentation, vol. 879 of AIP Conference Proceedings, pp. 792-795, June 2006.

[4] C. G. Schroer, O. Kurapova, J. Patommel et al., "Hard x-ray nanoprobe based on refractive x-ray lenses," Applied Physics Letters, vol. 87, no. 12, Article ID 124103, pp. 1-3, 2005.

[5] H. C. Kang, H. Yan, R. P. Winarski et al., "Focusing of hard $\mathrm{X}$-rays to 16 nanometers with a multilayer Laue lens," Applied Physics Letters, vol. 92, no. 22, Article ID 221114, 3 pages, 2008.

[6] G.-C. Yin, Y.-F. Song, M.-T. Tang et al., "30 nm resolution Xray imaging at $8 \mathrm{keV}$ using third order diffraction of a zone plate lens objective in a transmission microscope," Applied Physics Letters, vol. 89, no. 22, Article ID 221122, 3 pages, 2006.

[7] Y. Feng, M. Feser, A. Lyon et al., "Nanofabrication of high aspect ratio $24 \mathrm{~nm} \mathrm{x}$-ray zone plates for $\mathrm{x}$-ray imaging applications," Journal of Vacuum Science and Technology B, vol. 25, no. 6, pp. 2004-2007, 2007.

[8] Y. Suzuki and H. Toda, Advanced Tomographic Methods in Materials Research and Engineering, Oxford University Press, Oxford, UK, 2008, Section 7.1.

[9] Y. Suzuki, "Resolution limit of refractive lens and fresnel lens in X-ray region," Japanese Journal of Applied Physics. Part 1, vol. 43, no. 10, pp. 7311-7314, 2004.

[10] M. Sekimoto, A. Ozawa, T. Ohkubo, H. Yoshihira, M. Kakuchi, and T. Tamura, X-Ray Microscopy II, Springer, Berlin, Germany, 1988.

[11] A. Ozawa, T. Tamamura, T. Ishii, H. Yoshihara, and T. Kagoshima, "Application of X-ray mask fabrication technologies to high resolution, large diameter Ta Fresnel zone plates," Microelectronic Engineering, vol. 35, no. 1-4, pp. 525-529, 1997.

[12] D. Attwood, Soft X-Rays and Extreme Ultraviolet Radiation, Cambridge University Press, Cambridge, UK, 1999, Section 9.

[13] M. Born and E. Wolf, Principles of Optics, Cambridge University Press, Cambridge, UK, 1999.

[14] Y. Suzuki, K. Uesugi, N. Takimoto, et al., "Construction and commissioning of a $248 \mathrm{~m}$-long beamline with $\mathrm{x}$-ray undulator light source," in Synchrotron Radiation Instrumentation: 8th International Conference on Synchrotron Radiation Instrumentation, vol. 705 of AIP Conference Proceedings, pp. 344-347, 2004.

[15] Y. Suzuki, A. Takeuchi, H. Takano, T. Ohigashi, and H. Takenaka, "Diffraction-limited microbeam with Fresnel zone plate optics in hard X-ray regions," in X-Ray Micro- and NanoFocusing: Applications and Techniques II, Proceedings of the SPIE, pp. 74-84, San Diego, Calif, USA, July 2001.

[16] Y. Suzuki and F. Uchida, "Dark-field imaging in hard X-ray scanning microscopy," Review of Scientific Instruments, vol. 66, no. 2, pp. 1468-1470, 1995. 